\title{
Centerable Rotator for Measuring Properties of Crystals
}

\author{
Charles P. Saylor and Herbert B. Lowey
}

\author{
(February 23, 1965)
}

\begin{abstract}
By allowing the unsupported portion of a spindle to be relatively long so as to extend from the rim of the microscope stage all the way to the axis, simple controls can be provided for centering a crystal with respect to a horizontal axis of rotation. In the device which is described, centration can be assured within about a micron. The angle of rotation with respect to a fixed position can be read with an error not greater than 0.05 degree.
\end{abstract}

\section{Necessary Adjustments With the Single Axis Method}

In the single axis of rotation method of measuring optical properties $[1],{ }^{1}$ a crystal is held upon a spindle, it is rotated about an axis parallel to the stage of the microscope until one after another of the three characteristic vibration directions is made parallel to the stage, and each corresponding index of refraction of the crystal is then matched with that of an immersing liquid of known or subsequently determined refractivity. A crucial activity is the coordinated control of crystal orientation and polarizer vibration direction so as to achieve and recognize the three significant vibration directions. In this action, while the spindle is rotated slowly with one hand, the other hand, on the basis of information derived from changes in the interference figure, executes a controlled rotation of crossed polarizer and analyzer. The hand on the nicols sustains the condition by which an isogyre ${ }^{2}$ will always cross the center of the interference figure.

Devices for rotating the spindles have been described by Wood and Ayliffe [2], Bernal and Carlyle [3], Wilcox [4], Hartshorne and Swift [5], Hartshorne [6], and others. Well planned as these rotators are for relatively large crystals, they imperfectly cover one condition, important with crystals which cover less than the full microscopical field. If the interference figure of a small crystal is to be sufficiently contrasty, its image must be isolated from other light which might pass to the observer. This is best accomplished with a field diaphragm placed above the Bertrand lens at the level where the image of the crystal itself is formed or, if a Klein magnifier is used, by a diaphragm in the image plane of the eyepiece. Under these conditions, if the crystal is uncentered with respect to the axis of rotation, it is necessary continuously to adjust the crystal vertically or horizontally as its image strays from the position of the field diaphragm. Both hands and the mind, however, are already occupied with the control of two rotations. While foot

\footnotetext{
1 Figures in brackets indicate the literature references at the end of this paper 2 A locus of directions in a crystal (as seen in orthographic projection) for which the projected vibration directions, whether trivial or characteristic, are each parallel to the vibration directions of a polarizer or analyzer.
}

regulated motors might be installed, these would not overcome the problems that arise from division of attention. The effective solution is a rotator that permits centration of the crystal with respect to the axis of its rotation.

\section{Design of a Centerable Rotator}

In designing the apparatus which is described here, we found it expedient to depart from preceding practice to the extent of placing the entire rotator beyond the stage of the microscope. The actual support for the crystal, in the form of a small glass tube, thus extends unsupported across the radius of the stage. Because of the elasticity of glass and the smallness of the forces, this introduces no difficulty. Sensitive centering controls are easily achieved. In the device here described these operate at positions $90^{\circ}$ from each other. The adjusting of one control does not upset the centering that has been achieved with the other.

The centerable rotator which we are now using is illustrated in figure 1 . The stationary tube of the

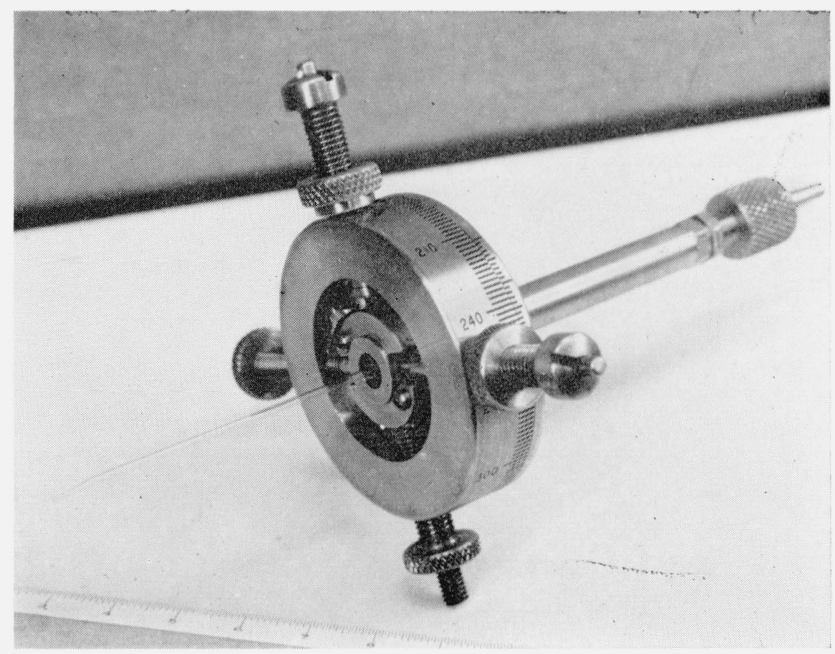

Figure 1. The centerable rotator.

The back and bottom nobs drive two jaws by reason of the difference between 28 and 32 thread screws. The others are spring loaded. The front and back jaws, cut away over half their diameters, lap over the top and bottom jaws. 
rotator, normally held in the chuck of a micromanipulator, is attached to an end plate with a 20 -interval vernier extending across $38^{\circ}$. At the right end, a thumb screw compresses a $2 \mathrm{~mm}$ long annulus of "microchemical" rubber tubing, $1.5 \mathrm{~mm}$ in internal and $3.5 \mathrm{~mm}$ in external diameter. This serves as a flexible chuck to hold and center the end of the tube farthest from the crystal. The hollow shaft has an internal diameter of about $3 \mathrm{~mm}$. Since the device was planned for use with $1.35 \pm 0.15$ mm glass tubing (a standard size), a total excursion of about $1 \frac{1}{2} \mathrm{~mm}$ at the controlled end is possible. The overhang of the tubing (the distance from the controlled end to the crystal) is not less than length of the rotator so that the range of adjustment at the crystal is about $3 \mathrm{~mm}$. In the drum at the left are the controllers, those with jaws driven by differential screws at the back and bottom and those with jaws pushed by light springs at the front and top. The sets which are separated by $90^{\circ}$ overlap like the parts of a lap joint.
Details of the centering controller are shown in figure 2. For movement of two jaws, a $1 / 4$-in.-28 machine screw is drilled along the center and tapped with a No. 8-32 thread. The actual jaws are cut into the central screws, one side of each of these being cut away for $11 \mathrm{~mm}$. They thus glide in a channel outside the point where they overlap the other set. This keeps them from rotating. The other two jaws are spring loaded. The differential movement is such that the jaw advances a little more than 0.1 $\mathrm{mm}$ for each full turn of the larger screw. This corresponds to about $0.2 \mathrm{~mm}$ at the crystal, thus providing all necessary sensitivity. By comparison, one full turn of the fine adjustment of the standard microscope produces a movement of $0.1 \mathrm{~mm}$.

In order to insert the glass tube into the rotator, it is convenient to have means of retracting both the screw driven and the spring loaded jaws. As we have chosen to manage this, the spring-loaded jaws are momentarily held back by a flat collar in the back of the jaws with two camlike wedges that can press the jaws outward.

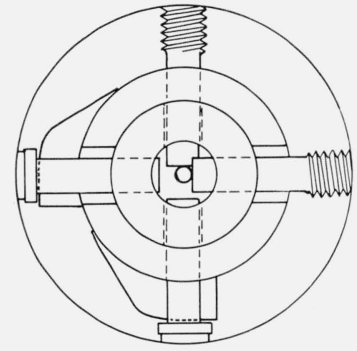

(a)

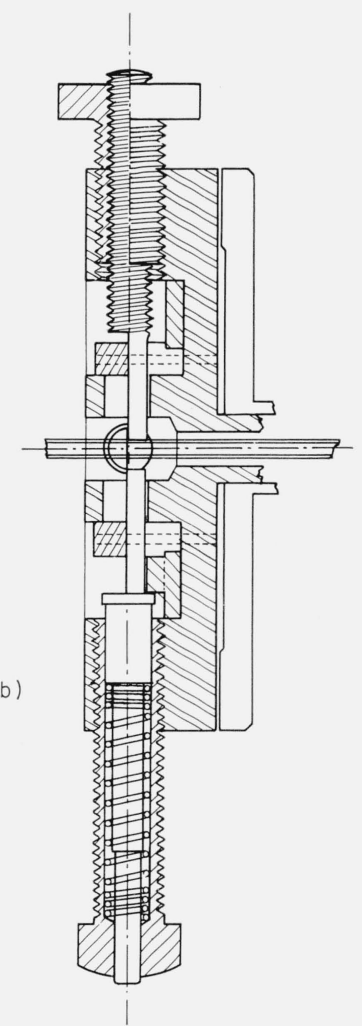

Figure 2. Details of the rotator.

(a) Overlap of centering jaws as seen from end. (b) Differential screw drive. (c) The clamp and aspirator. 
The graduated drum provides an easy means for recording positions of the spindle. Since the drum is only $5 \mathrm{~cm}$ in diameter, markings at $1 \mathrm{deg}$ intervals would be separated by less than $0.5 \mathrm{~mm}$. This would be too close. When $2 \mathrm{deg}$ marks are combined with the 20 interval vernier there is adequate accuracy. Thus the position of the drum as seen in figure 1 clearly lies between 213.5 and $213.6^{\circ}$, being closer to the former. Obviously the uncertainties are less than $0.05 \mathrm{deg}$. Since the accuracy of settings of the crystal is rarely better than $1 \mathrm{deg}$, this precision of measurement meets all requirements.

\section{Supporting the Crystals}

It is well to insert a word about the use that is made here of glass tubing for the spindle. Equipment is available for drawing tips on capillary glass tubing with practically perfect control. By having a sharply tapered end on such a tubular spindle, crystals can be held in either of two satisfactory ways. This can be by suction applied to the tubing by means of the nipple shown at the right, figure 1 . Although some immersion fluid leaks between the crystal and the end of the tube, the steadily increasing diameter prevents viscosity losses from destroying the force at the attachment. Occasionally the leakage is so great that too much of the immersion compound will be lost.

The second technique, which is also limited to tubes, makes use of drying cements. If a tapered very fine rod is inserted into a cement and withdrawn, surface tension tends to remove the cement down to such thinness that the residue cannot provide adhesion. With a somewhat larger rod, the residual cement adheres to the sides, but not to the end.
With a tapered tube, however, the outside is left nearly bare whereas the cement flowing into the canal forms a nearly plane surface across the end. When this plane end is touched to a crystal, the crystal is caught up by capillarity. As the cement dries in a circle of contact between the crystal and the tube, the attachment becomes very strong. Of the many different cements which can be used, one of the most satisfactory is ordinary corn syrup.

By either of these methods of attachment, a crystal will hold an unvarying position at the end of a tubular spindle. The attachment endures through the necessary reorientations of the crystal, changes in composition of the immersing fluid, and determinations of the refractive index of the fluid. Since the correctness of orientation of the crystals and their vibration directions can be found with adequate sensitivity, and since the accuracy of a refractive index measurement is limited only by the criterion of match and the patience of the observer, the rotator which is here described facilitates highly accurate determinations of the principal optical properties of crystals.

\section{References}

[1] C. P. Saylor, Chemical Society of Washington, Spring Meeting, May 11, 1944.

J. L. Rosenfeld, Am. Mineral. 35, 902 (1950).

R. E. Wilcox, Am. Mineral. 44, 1272 (1959).

[2] R. G. Wood and S. G. Ayliffe, J. Sci. Instr. 12, 194 (1935),

[3] J. D. Bernal and C. H. Carlisle, J. Sci. Instr. 24, 107 (1947).

[4] R. E. Wilcox, Am. Mineral. 44, 1272 (1959).

[5] N. H. Hartshorne and P. M. Swift, J. Roy. Mic. Soc. 75 , 129 (1955).

[6] N. H. Hartshorne, Mineral. Mag. 33, 693 (1963).

(Paper 69C3-198) 